\title{
¿Es más difícil la colecistectomía laparoscópica después de una colangiopancreatografía retrógrada endoscópica? Experiencia en un hospital de tercer nivel
}

\author{
Is the laparoscopic cholecystectomy more difficult after endoscopic \\ retrograde cholangiopancreatography? Experience in a third level hospital
}

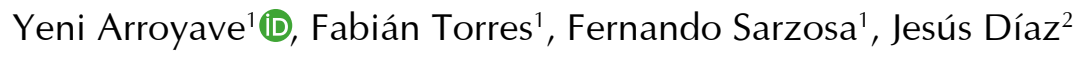
Médico, especialista en cirugía general, Universidad del Cauca, Popayán, Colombia
Médico, especialista en Cirugía general, Gastroenterología y Endoscopia Digestiva y en Epidemiología; profesor, Departamento
de Ciencias Quirúrgicas, Universidad del Cauca, Popayán, Colombia

En memoria de nuestro gran amigo Fabián Torres (Q.E.P.D), quién nos enseñó en vida que nada es imposible, y en su partida, que debemos luchar hasta el final.

\section{Resumen}

Introducción. La colecistectomía laparoscópica es el tratamiento estándar para la colecistitis aguda. En pacientes con coledocolitiasis, la colangiopancreatografía retrógrada endoscópica es el tratamiento de elección. Se ha reportado que, después de este procedimiento endoscópico, la colecistectomía laparoscópica es más difícil y son mayores las tasas de conversión, hemorragia y tiempo operatorio. El objetivo de este estudio fue determinar si en nuestro medio las colecistectomías laparoscópicas posteriores a este procedimiento endoscópico presentan más complicaciones posquirúrgicas y mayor dificultad técnica.

Métodos. Estudio de cohorte prospectivo, en el que se comparó un grupo de pacientes sometidos a colecistectomía laparoscópica previa colangiopancreatografía retrógrada endoscópica, contra un grupo homogéneo de pacientes sin colangiografía previa, para evaluar la dificultad en la colecistectomía laparoscópica, la conversión, la reintervención y las complicaciones.

Resultados. El 45,4 \% de las cirugías fueron difíciles. No hay relación entre la realización previa de colangiopancreatografía retrógrada endoscópica y la dificultad de la colecistectomía laparoscópica. Con el modelo de regresión logística, se encontraron como factores predictores para una cirugía difícil, la edad, el sexo masculino, la cirugía abdominal previa, la colecistitis aguda y la mayor gravedad de la colecistitis aguda.

Fecha de recibido: 21/06/2019 - Fecha de aceptación: 25/02/2020

Correspondencia: Yeni Arroyave, Carrera 6 \# 10 Norte -143 Hospital Universitario San José, Departamento de Ciencias Quirúrgicas, tercer piso, Popayán, Colombia. Teléfono: 3012632778

Correo electrónico: yarroyave@unicauca.edu.co

Citar como: Arroyave Y, Torres F, Sarzosa F, Díaz J. ¿Es más difícil la colecistectomía laparoscópica después de una colangiopancreatografía retrógrada endoscópica? Experiencia en un hospital de tercer nivel. Rev Colomb Cir. 2020;35:436-48. https://doi.org/10.30944/20117582.781

Este es un artículo de acceso abierto bajo una Licencia Creative Commons - BY-NC-ND https://creativecommons.org/licenses/by-ncnd/4.0/deed.es 
Conclusión. La colangiopancreatografía retrógrada endoscópica en nuestro medio no constituye un factor de riesgo para dificultad en la colecistectomía laparoscópica. Debe prestarse especial cuidado al sexo masculino, la gravedad de la colecistitis aguda, los antecedentes de cirugía abdominal y la presencia de comorbilidades a la hora de planear una colecistectomía laparoscópica, tomando precauciones adicionales en estos casos para prevenir complicaciones.

Palabras clave: colecistitis aguda; colangiopancreatografía retrógrada endoscópica; colecistectomía laparoscópica; complicaciones; conversión a cirugía abierta.

\begin{abstract}
Introduction: Laparoscopic cholecystectomy is the standard treatment for acute cholecystitis. In patients who also have choledocholithiasis, endoscopic retrograde cholangiopancreatography is the treatment of choice. In some studies, it has been reported that, after this endoscopic examination, laparoscopic cholecystectomy is more difficult, and conversion rates, bleeding and operative time are higher. The objective of this study was to determine whether laparoscopic cholecystectomies after this endoscopic procedure present more postoperative complications and greater technical difficulty in our setting.

Methods: Prospective cohort study, in which a group of patients who underwent laparoscopic cholecystectomy prior endoscopic retrograde cholangiopancreatography was compared against a homogeneous group of patients without previous cholangiography, to assess the difficulty of laparoscopic cholecystectomy, conversion, reoperation and complications.

Results: $45.4 \%$ of the surgeries were difficult. There is no relationship between the previous performance of ERCP and the difficulty of laparoscopic cholecystectomy. With the logistic regression model, age, male gender, previous abdominal surgery, acute cholecystitis and greater degree of severity of acute cholecystitis were found as predictive factors for difficult surgery.

Conclusion: ERCP in our setting is not a risk factor for difficult laparoscopic cholecystectomy. Special care should be taken to the male gender, the severity of acute cholecystitis, the history of abdominal surgery and the presence of comorbidities when planning a laparoscopic cholecystectomy, taking additional precautions in these cases to prevent complications.
\end{abstract}

Key words: cholecystitis, acute; cholangiopancreatography, endoscopic retrograde; cholecystectomy, laparoscopic; complications; conversion to open surgery.

\section{Introducción}

La colecistectomía laparoscópica es el procedimiento de elección para el tratamiento de la colecistitis aguda ${ }^{\mathrm{I}}$. En pacientes con coledocolitiasis coexistente, la colangiopancreatografía retrógrada endoscópica (CPRE) con esfinterotomía, es el tratamiento de elección; llevar a cabo la colecistectomía laparoscópica en un intervalo corto después de la CPRE ayuda a evitar la recurrencia de síntomas biliares ${ }^{2}$. En algunos estu- dios se ha reportado que, después de la CPRE, la colecistectomía laparoscópica es más difícil, con mayores tasas de conversión, hemorragia y tiempo operatorio ${ }^{3,4}$. Se cree que esto se debe a la inflamación y posterior fibrosis de las estructuras del ligamento hepatoduodenal, por instrumentación de la vía biliar, como efecto directo del medio de contraste, o por la colonización bacteriana de la vía biliar posterior a la papilotomía, lo cual dificulta la disección del triángulo de Calot ${ }^{4}$. 
En el Hospital Universitario San José de Popayán, Colombia, en el año 2016 se practicaron 320 colecistectomías laparoscópicas no ambulatorias, de las cuales 72 (22,5\%) fueron en pacientes con CPRE previa. Esto refleja, no solo la frecuencia de la enfermedad biliar en nuestro medio, sino también, que un buen número de pacientes se presentan con obstrucción del conducto biliar común. Poder predecir la dificultad técnica en estos casos, facilitaría la planeación quirúrgica, incluyendo la necesidad de que estos pacientes fueran operados por los cirujanos con mayor experiencia.

Este estudio tuvo como objetivo determinar, si en nuestro medio las colecistectomías laparoscópicas practicadas después de CPRE presentan más complicaciones posquirúrgicas y mayor dificultad técnica, que las que se practican sin ese antecedente.

\section{Métodos}

Se trata de un estudio observacional prospectivo de cohorte. Se incluyeron todos los pacientes con enfermedad litiásica biliar a quienes se les practicó colecistectomía laparoscópica, desde el $I^{\circ}$ de enero hasta el 3I de diciembre de 2017. Los pacientes que cumplieron con los criterios de inclusión se dividieron en dos grupos: 'con CPRE', a los que se les practicó una colangiopancreatografía retrógrada endoscópica antes de la colecistectomía laparoscópica y 'sin CPRE' a los que no se le practicó este procedimiento endoscópico previo a la cirugía.

\section{Criterios de exclusión}

- Pacientes en quienes se practicó colecistectomía laparoscópica de manera electiva (ambulatoria)

- Pacientes sometidos a colecistectomía laparoscópica por una causa diferente a litiasis o pancreatitis de origen biliar.

\section{Tamaño de la muestra}

Teniendo en cuenta el número de colecistectomías laparoscópicas previo CPRE hechas en el
Hospital Universitario San José de Popayán en el año inmediatamente anterior (2016), con un intervalo de confianza de $95 \%\left(\mathrm{IC}_{95} \%\right.$ ) y un error máximo permitido de $3,5 \%$, se calculó un tamaño de la muestra necesario de 67 pacientes con colecistectomía laparoscópica posterior a CPRE. Para el grupo control, sin la intervención (CPRE), se incluyeron todos los pacientes operados en el mismo periodo.

\section{Recolección de los datos}

Tres de los investigadores recolectaron la información semanalmente, revisando las historias clínicas de los pacientes sometidos a colecistectomía laparoscópica durante esa semana. Los pacientes se siguieron hasta su egreso, muerte o remisión.

Se usó un instrumento de recolección de la información diseñado por el grupo de investigación, el cual incluía, además de los datos sociodemográficos, las variables descritas previamente que están asociadas a la dificultad quirúrgica, y las variables que permitieran establecer si se trató de una colecistectomía difícil o no. También, se registraron las cirugías reportadas por los cirujanos en la descripción operatoria como "difíciles".

El instrumento se dividió en seis secciones: datos de identificación, variables prequirúrgicas, que incluían el índice de masa corporal (IMC), clasificación de la American Society of Anesthesiologists (ASA), alguna cirugía abdominal previa y comorbilidades; diagnóstico prequirúrgico según las guías de Tokio $2013{ }^{5}$ y la revisión de Atlanta $2012{ }^{6}$ para los pacientes con pancreatitis aguda; datos de la CPRE, entre ellos, cantidad de procedimientos antes de la colecistectomía laparoscópica, tiempo entre la última CPRE y la cirugía, práctica de papilotomía o colocación de endoprótesis (stent), confirmación de coledocolitiasis y si se pudo resolver mediante dicho procedimiento, presencia y drenaje de colangitis; hallazgos quirúrgicos, complicaciones y años de experiencia del cirujano. Se usaron las guías de Tokio 2013 porque, en el tiempo de planeación del estudio y recolección de la información, no habían sido publicadas aún las guías del 2018. 


\section{Colecistectomía laparoscópica difícil}

Se consideraron difíciles todas las colecistectomías laparoscópicas que incluyen uno o más de los siguientes criterios descritos en la literatura científica ${ }^{7,8}$ :

I) adherencias densas (firmes),

2) vesícula biliar contraída, fibrótica o ambas (escleroatrófica),

3) vesícula gangrenosa,

4) síndrome de Mirizzi,

5) hemorragia: conversión por hemorragia,

6) plastrón,

7) elementos del triángulo de Calot no visualizados y

8) fístula bilioentérica.

Dado que en la mayoría de las descripciones operatorias no se cuantificó el sangrado, se consideró significativa la hemorragia que generó necesidad de conversión.

Para el estudio, se consideró la colecistectomía laparoscópica reportada como difícil cuando en la nota operatoria el cirujano concluía que el procedimiento fue "difícil", o cuando usaba términos como "dificultad", "laborioso" o sus sinónimos, para describir algún momento de la disección.

\section{Análisis estadístico}

El análisis estadístico se llevó a cabo con el programa estadístico SPSS ${ }^{\mathrm{TM}}$ (IBM), versión 20. Las variables cualitativas se expresaron como frecuencias y proporciones (\%), y las variables cuantitativas, con su mediana, y valor mínimo y máximo, ya que se comprobó con la prueba de Kolmogorov-Smirnov que no tenían una distribución normal.

Las variables cualitativas se compararon con la prueba de $c^{2} y$, las variables cuantitativas, mediante la prueba no paramétrica $U$ de Mann-Whitney dado que su distribución no era normal. Para evaluar la relación entre colecistectomía difícil y los factores de riesgo propuestos en la literatura, entre ellos una CPRE previa, se hizo un análisis multivariado por medio de regresión logística binaria. Se consideró un valor de $\mathrm{p}$ menor de 0,05 como estadísticamente significativo.

\section{Resultados}

Entre el $\mathrm{I}^{\circ}$ de enero y el 3I de diciembre de 20I7, 500 pacientes fueron sometidos a colecistectomía laparoscópica en el Hospital Universitario San José, en Popayán, Colombia. Después de usar los criterios de exclusión, se seleccionaron 342 , a 67 de los cuales se les había practicado la CPRE previo a la cirugía (figura I).

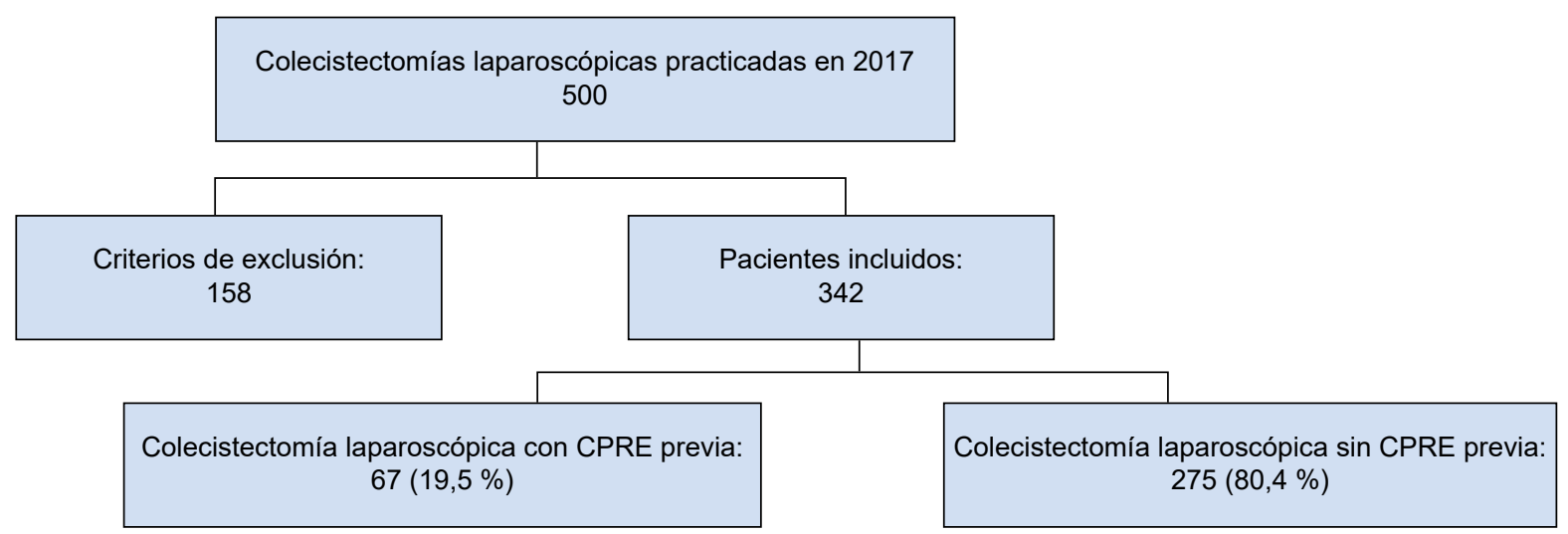

Figura 1. Distribución de los pacientes incluidos en el estudio. 
La mediana de edad fue de 53 años y el rango de 15 a 9o. En ambas cohortes ('con CPRE' y 'sin CPRE'), la mayoría de los pacientes fueron de sexo femenino, clasificados como ASA I y II y de procedencia rural. La presencia de comorbilidades fue proporcional entre ambos grupos; el $66 \%$ tenía hipertensión arterial sistémica, y otras comorbilidades incluyeron diabetes mellitus, enfermedad renal crónica, asma, enfermedad pulmonar obstructiva (EPOC) e insuficiencia cardiaca congestiva, entre otras.

Las características sociodemográficas y clínicas prequirúrgicas de cada grupo se muestran en la tabla I. Consideramos importante hacer énfasis en los siguientes puntos:

- En la cohorte 'sin CPRE', a dos pacientes se les hizo el diagnóstico prequirúrgico de colangitis por presentar un patrón colestásico asociado a una reacción inflamatoria sistémica; a ambos se les practicó la colangiografía intraoperatoria sin observarse defectos de llenado, por lo cual se continuó con los pasos usuales de la colecistectomía laparoscópica.

- Un paciente con pancreatitis y sin diagnóstico de colelitiasis, se sometió a colecistectomía laparoscópica, pues el equipo quirúrgico consideró muy probable un origen biliar por el cuadro clínico y los exámenes paraclínicos.

- A seis pacientes que cumplían con los criterios para coledocolitiasis o colangitis, aunque sin diagnóstico prequirúrgico de colelitiasis (la ecografía no mostró cálculos vesiculares), se les practicó la CPRE.

- A cuatro pacientes con pancreatitis aguda y riesgo intermedio para coledocolitiasis, según los predictores clínicos de la American Society for Gastrointestinal Endoscopy (ASGE) 9 , se les practicó la colangiopancreatografía retrógrada endoscópica; en todos se confirmó la presencia de coledocolitiasis y, en uno, fue necesaria una endoprótesis biliar por no lograrse resolver completamente la litiasis biliar.
Tabla 1. Características sociodemográficas y clínicas prequirúrgicas de los pacientes sometidos a colecistectomía laparoscópica.

\begin{tabular}{|c|c|c|}
\hline Variables & $\begin{array}{l}\text { Con CPRE }(n=67) \\
\text { Frecuencia (\%) }\end{array}$ & $\begin{array}{c}\text { Sin CPRE }(n=275) \\
\text { Frecuencia (\%) }\end{array}$ \\
\hline $\begin{array}{l}\text { Edad (años), mediana } \\
\text { (mínimo-máximo) }\end{array}$ & $55(18-83)$ & $52(15-90)$ \\
\hline $\begin{array}{l}\text { Sexo } \\
\text { Femenino } \\
\text { Masculino } \\
\end{array}$ & $\begin{array}{l}36(53,7) \\
31(46,3)\end{array}$ & $\begin{array}{l}172(62,5) \\
103(37,5)\end{array}$ \\
\hline $\begin{array}{l}\text { Procedencia } \\
\text { Rural } \\
\text { Urbana }\end{array}$ & $\begin{array}{l}44(65,7) \\
23(34,3)\end{array}$ & $\begin{array}{l}140(50,9) \\
135(49,1)\end{array}$ \\
\hline $\begin{array}{l}\text { Comorbilidad médica } \\
\text { Sí } \\
\text { No }\end{array}$ & $\begin{array}{l}17(25,4) \\
50(74,6) \\
\end{array}$ & $\begin{array}{r}71(25,8) \\
204(74,2) \\
\end{array}$ \\
\hline $\begin{array}{l}\text { Índice de Masa Corpo } \\
\text { Bajo peso } \\
\text { Peso normal } \\
\text { Sobrepeso } \\
\text { Obesidad }\end{array}$ & $\begin{array}{c}0(0) \\
27(40,3) \\
25(37,3) \\
15(22,4)\end{array}$ & $\begin{array}{r}3(1,1) \\
115(41,8) \\
105(38,2) \\
52(18,9)\end{array}$ \\
\hline $\begin{array}{l}\text { Clasificación preanes } \\
\text { ASA I } \\
\text { ASA II } \\
\text { ASA III } \\
\text { ASA IV }\end{array}$ & $\begin{array}{l}\text { tésica } \\
\qquad \begin{array}{c}11(16,4) \\
47(70,1) \\
9(13,4) \\
0(0)\end{array}\end{array}$ & $\begin{array}{r}81(29,5) \\
165(60,0) \\
28(10,2) \\
1(0,4)\end{array}$ \\
\hline $\begin{array}{l}\text { Cirugía abdominal pre } \\
\text { Sí } \\
\text { No }\end{array}$ & $\begin{array}{r}2(3) \\
\text { evia } \\
65(97) \\
\end{array}$ & $\begin{array}{r}20(7,3) \\
255(92,7) \\
\end{array}$ \\
\hline $\begin{array}{l}\text { Colelitiasis } \\
\text { Sí } \\
\text { No } \\
\end{array}$ & $\begin{array}{r}\text { óstico prequirúrgi } \\
61(91) \\
6(9) \\
\end{array}$ & $\begin{array}{r}274(99,6) \\
1(0,4) \\
\end{array}$ \\
\hline $\begin{array}{l}\text { Colecistitis } \\
\text { No } \\
\text { Sí } \\
\text { Grado } 1 \\
\text { Grado } 2 \\
\text { Grado } 3 \\
\end{array}$ & $\begin{array}{r}46(68,7) \\
21(31,3) \\
14(66,6) \\
6(28,6) \\
1(4,8) \\
\end{array}$ & $\begin{array}{r}81(29,5) \\
194(70,5) \\
104(53,6) \\
83(42,8) \\
7(3,6) \\
\end{array}$ \\
\hline $\begin{array}{l}\text { Coledocolitiasis } \\
\text { Sí } \\
\text { No } \\
\end{array}$ & $\begin{array}{r}63(94) \\
4(6) \\
\end{array}$ & $\begin{array}{r}5(1,8) \\
270(98,2) \\
\end{array}$ \\
\hline $\begin{array}{l}\text { Colangitis } \\
\text { No } \\
\text { Sí } \\
\text { Grado } 1 \\
\text { Grado } 2 \\
\text { Grado } 3\end{array}$ & $\begin{array}{r}43(64,2) \\
24(35,8) \\
9(37,5) \\
13(54,2) \\
2(8,3)\end{array}$ & $\begin{array}{c}273(99,3) \\
2(0,7) \\
1(50) \\
1(50) \\
0(0)\end{array}$ \\
\hline $\begin{array}{l}\text { Pancreatitis } \\
\text { No } \\
\mathrm{Si} \\
\text { Leve } \\
\text { Moderadamente } \\
\text { severa } \\
\end{array}$ & $\begin{array}{r}54(80,6) \\
13(19,4) \\
12(92,3) \\
1(7,7)\end{array}$ & $\begin{array}{l}253(92) \\
22(8) \\
17(77,3) \\
5(22,7)\end{array}$ \\
\hline Total & 67 & 275 \\
\hline
\end{tabular}

CPRE: colangiopancreatografía retrógrada endoscópica; ASA: clasificación de la American Society of Anesthesiologists 
Los cirujanos que practicaron las colecistectomías laparoscópicas fueron $\mathbf{1} 7$, de los cuales cinco tenían menos de cinco años de experiencia, uno, de 5 a Io años, cuatro, de Io a 20 años, y siete, más de 20 años de experiencia.

De las 342 colecistectomías laparoscópicas incluidas en el estudio, 155 (45,3\%) cumplieron los criterios para una cirugía difícil; sin embargo, solo $70(45,2 \%)$ de estas fueron descritas como difíciles por los cirujanos. Los criterios que usaron para describirlas como "difíciles" fueron la presencia de inflamación, fibrosis del triángulo de Calot, adherencias, plastrón, síndrome de Mirizzi, vesícula empotrada en el lecho quirúrgico o sangrado intraoperatorio.

La CPRE previa, la papilotomía o la colocación de endoprótesis biliar, no se asociaron con dificultad en la colecistectomía laparoscópica o conversión a cirugía abierta (tabla 2). Las variables de sexo, edad, colecistitis aguda y gravedad de la colecistitis aguda se asociaron con una colecistectomía laparoscópica difícil de forma estadísticamente significativa. Al comparar los dos grupos de estudio se encontró que, 15 (22\%) de las colecistectomías laparoscópicas del grupo 'con CPRE' y 55 (20 \%) de las colecistectomías del grupo 'sin CPRE', fueron reportadas por los cirujanos como difíciles.

La tasa global de conversión a cirugía abierta fue de $5,5 \%$. En cuanto a la conversión a cirugía abierta, el sexo y la percepción del cirujano de la cirugía como difícil, fueron las variables con asociación estadísticamente significativa. En la cohorte 'con CPRE', el Ioo \% de las causas de conversión a cirugía abierta estuvieron asociadas con dificultad en la disección o falta de visualización de los elementos del triángulo de Calot. En la cohorte 'sin CPRE', las causas de conversión fueron: plastrón (56,2\%), sangrado (I8,8 \%), adherencias (I8,8\%) y difícil disección del triángulo de Calot (6,2 \%).

En el grupo 'con CPRE', la mediana de tiempo entre el procedimiento endoscópico y la colecistectomía laparoscópica fue de cuatro días (rango: I-I6); no hubo asociación estadísticamente significativa entre este intervalo de tiempo y la difi- cultad de la intervención laparoscópica $(\mathrm{p}=0,78)$. Además, la mediana del tiempo quirúrgico fue de 70 minutos (rango: 30-193), en comparación con 65 minutos (rango: 22-I80) en el grupo 'sin CPRE'; una diferencia que no fue estadísticamente significativa ( $\mathrm{p}=\mathrm{O}, \mathrm{o82})$.

En el grupo 'con CPRE' no hubo complicaciones intraoperatorias y, en el otro grupo, tres pacientes presentaron sangrado, dos pacientes lesión intestinal, y uno, lesión de la vía biliar.

No hubo reintervenciones dentro de las primeras 24 horas en ningún caso. En el grupo 'con CPRE', tres $(4,5 \%)$ pacientes requirieron reintervención después de las 24 horas, todos por colección intraabdominal, y en el grupo 'sin CPRE', I7 $(6,3 \%)$ pacientes necesitaron reintervenciones, I3 pacientes una vez, un paciente dos veces y tres pacientes en tres ocasiones. Las causas se muestran en la tabla 3. Vale la pena aclarar que, en el caso de colecciones intraabdominales, algunas se resolvieron mediante drenaje percutáneo; sin embargo, se consideraron como reintervenciones, dado que correspondían a una intervención adicional al procedimiento original.

La CPRE previa a la colecistectomía laparoscópica, tampoco se asoció con reintervención después de las 24 horas o complicaciones posoperatorias. La edad, la presencia de alguna comorbilidad, el diagnóstico prequirúrgico de colecistitis aguda y una mayor gravedad de la colecistitis aguda, se asociaron de manera significativa con la necesidad de reintervención después de las 24 horas de la colecistectomía laparoscópica. Las comorbilidades, la colecistitis aguda y su gravedad, se asociaron también con complicaciones posoperatorias (tabla 4).

En total, $23(8,4 \%)$ pacientes de los 275 del grupo 'sin CPRE' tuvieron complicaciones posoperatorias, de los cuales, Io presentaron complicaciones médicas no abdominales (como infarto agudo de miocardio, tromboembolia pulmonar, neumonía, falla respiratoria, falla renal y descompensación de diabetes, entre otras), asociadas o no a complicaciones abdominales en 15 pacientes (como colección intraabdominal, hemoperitoneo, íleo adinámico y biliperitoneo por fuga 
Tabla 2. Variables asociadas con cirugía difícil o conversión a cirugía abierta.

\begin{tabular}{|c|c|c|c|c|c|c|c|}
\hline \multirow[b]{2}{*}{ Variables } & \multicolumn{3}{|c|}{ Cirugía difícil } & \multicolumn{3}{|c|}{ Conversión } & \multirow{3}{*}{$\begin{array}{r}\text { Total } \\
342\end{array}$} \\
\hline & Sí (\%) & No (\%) & \multirow[t]{2}{*}{ p } & Sí (\%) & No (\%) & \multirow[t]{2}{*}{ p } & \\
\hline Total & 155 & 187 & & 19 & 323 & & \\
\hline$\overline{\text { CPRE }}$ & & & 0,517 & & & 0,668 & \\
\hline Sí & $28(41,8)$ & $39(58,2)$ & & $3(4,5)$ & $64(95,5)$ & & 67 \\
\hline No & $127(46,2)$ & $148(53,8)$ & & $16(5,8)$ & $259(94,2)$ & & 275 \\
\hline Papilotomía & & & 0,517 & & & 0,668 & \\
\hline Sí & $28(41,8)$ & $39(58,2)$ & & $3(4,5)$ & $64(95,5)$ & & 67 \\
\hline No & $127(46,2)$ & $148(53,8)$ & & $16(5,8)$ & $259(94,2)$ & & 275 \\
\hline Endoprótesis & & & 0,698 & & & 0,748 & \\
\hline Sí & $3(33,3)$ & $6(66,7)$ & & $0(0)$ & $9(100)$ & & 9 \\
\hline No & $25(43,1)$ & $33(56,9)$ & & $3(5,2)$ & $55(94,8)$ & & 58 \\
\hline Sexo & & & 0,001 & & & 0,028 & \\
\hline Masculino & $76(56,7)$ & $58(43,3)$ & & $12(9)$ & $122(91)$ & & 134 \\
\hline Femenino & $79(38)$ & $129(62)$ & & $7(3,4)$ & $201(96,6)$ & & 208 \\
\hline Edad (años) & & & 0,000 & & & 0,092 & \\
\hline $15-20$ & $4(28,6)$ & $10(71,4)$ & & $0(0)$ & $14(100)$ & & 14 \\
\hline $21-40$ & $29(29,9)$ & $68(70,1)$ & & $1(1)$ & $96(99)$ & & 97 \\
\hline $41-60$ & $42(42,9)$ & $56(57,1)$ & & $6(6,1)$ & $92(93,9)$ & & 98 \\
\hline $61-80$ & $70(60,3)$ & $46(39,7)$ & & $10(8,6)$ & $106(91,4)$ & & 116 \\
\hline$>80$ & $10(58,8)$ & $7(41,2)$ & & $2(11,8)$ & $15(88,2)$ & & 17 \\
\hline Cirugía abdominal previa & & & 0,121 & & & 0,228 & \\
\hline Sí & $14(60,9)$ & $9(39,1)$ & & $0(0)$ & $23(100)$ & & 23 \\
\hline No & $141(44,2)$ & $178(55,8)$ & & $19(6)$ & $300(94)$ & & 319 \\
\hline Comorbilidad & & & 0,129 & & & 0,548 & \\
\hline Sí & $46(52,3)$ & $42(47,7)$ & & $6(6,8)$ & $82(93,2)$ & & 254 \\
\hline No & $109(42,9)$ & $145(57,1)$ & & $13(5,1)$ & $241(94,9)$ & & 88 \\
\hline ASA & & & 0,196 & & & 0,855 & \\
\hline 1 & $39(42,4)$ & $53(57,6)$ & & $4(4,3)$ & $88(95,7)$ & & 92 \\
\hline II & $93(43,9)$ & $119(56,1)$ & & $12(5,7)$ & $200(94,3)$ & & 212 \\
\hline III & $22(59,5)$ & $15(40,5)$ & & $3(8,1)$ & $34(91,9)$ & & 37 \\
\hline IV & $1(100)$ & $0(0)$ & & $0(0)$ & $1(100)$ & & 1 \\
\hline IMC & & & 0,833 & & & 0,900 & \\
\hline Bajo peso & $2(66,7)$ & $1(33,3)$ & & $0(0)$ & $3(100)$ & & 3 \\
\hline Peso normal & $62(43,7)$ & $80(56,3)$ & & $9(6,3)$ & $133(93,7)$ & & 142 \\
\hline Sobrepeso & $59(45,4)$ & $71(54,6)$ & & $6(4,6)$ & $124(95,4)$ & & 130 \\
\hline Obesidad & $32(47,8)$ & $35(52,2)$ & & $4(6,0)$ & $63(94)$ & & 67 \\
\hline Colecistitis & & & 0,000 & & & 0,978 & \\
\hline No & $36(28,3)$ & $91(71,7)$ & & $7(5,5)$ & $120(94,5)$ & & 127 \\
\hline Sí & $119(55,3)$ & $96(44,7)$ & & $12(5,6)$ & $203(94,4)$ & & 215 \\
\hline Grado 1 & $52(44,1)$ & $66(55,9)$ & 0,001 & $5(4,2)$ & $113(95,8)$ & 0,714 & 118 \\
\hline Grado 2 & $61(68,5)$ & $28(31,5)$ & & $6(6,7)$ & $83(93,3)$ & & 89 \\
\hline Grado 3 & $6(75)$ & $2(25)$ & & $1(12,5)$ & $7(87,5)$ & & 8 \\
\hline Colangitis & & & 0,618 & & & 0,692 & \\
\hline No & $142(44,9)$ & $174(55,1)$ & & $18(5,7)$ & $298(94,3)$ & & 316 \\
\hline Sí & $13(50)$ & $13(50)$ & & $1(3,8)$ & $25(96,2)$ & & 26 \\
\hline Grado 1 & $6(60)$ & $4(40)$ & 0,816 & $0(0)$ & $10(100)$ & 0,853 & 10 \\
\hline Grado 2 & $6(42,9)$ & $8(57,1)$ & & $1(7,1)$ & $13(92,9)$ & & 12 \\
\hline Grado 3 & $1(50)$ & $1(50)$ & & $0(0)$ & $2(100)$ & & 2 \\
\hline Pancreatitis & & & 0,216 & & & 0,462 & \\
\hline No & $143(46,6)$ & $164(53,4)$ & & $18(5,9)$ & $289(94,1)$ & & 307 \\
\hline Sí & $12(34,2)$ & $23(65,7)$ & & $1(2,9)$ & $34(97,1)$ & & 35 \\
\hline Leve & $9(31)$ & $20(69)$ & 0,267 & $1(3,4)$ & $28(96,6)$ & 0,721 & 29 \\
\hline Moderadamente severa & $3(50)$ & $3(50)$ & & $0(0)$ & $6(100)$ & & 6 \\
\hline Reportada como difícil & & & 0,000 & & & 0,000 & \\
\hline Sí & $70(100)$ & $0(0)$ & & $11(15,7)$ & $59(84,3)$ & & 70 \\
\hline No & $85(31,3)$ & $187(68,8)$ & & $8(2,9)$ & $264(97,1)$ & & 272 \\
\hline
\end{tabular}

CPRE: colangiopancreatografía retrógrada endoscópica; ASA: clasificación de la American Society of Anesthesiologists; IMC: índice de masa corporal. 
Tabla 3. Distribución porcentual de las causas de reintervención después de 24 horas, en pacientes sometidos a colecistectomía laparoscópica sin colangiopancreatografía retrógrada endoscópica previa

\begin{tabular}{lcc}
\hline Causa & Frecuencia & Porcentaje \\
\hline Colección intraabdominal & 8 & 47,1 \\
\hline Desempaquetamiento & 4 & 23,5 \\
\hline Sangrado del lecho & 2 & 11,8 \\
\hline Fuga biliar o biliperitoneo & 2 & 11,8 \\
\hline Sangrado de pared abdominal & 1 & 5,9 \\
\hline Total & 17 & 100 \\
\hline
\end{tabular}

Tabla 4. Asociación de variables con reintervención después de 24 horas y complicaciones posoperatorias

\begin{tabular}{|c|c|c|c|c|c|c|c|}
\hline \multirow[t]{2}{*}{ Variables } & \multicolumn{3}{|c|}{ Reintervención $>\mathbf{2 4}$ horas } & \multicolumn{3}{|c|}{ Complicación posoperatoria } & \multirow{3}{*}{$\begin{array}{l}\text { Tota } \\
342\end{array}$} \\
\hline & Sí (\%) & No (\%) & $\mathbf{P}$ & Sí & No & $\mathbf{p}$ & \\
\hline Total & 20 & 322 & & 27 & 315 & & \\
\hline CPRE & & & 0,594 & & & 0,515 & \\
\hline Sí & $3(4,5)$ & $64(95,5)$ & & $4(6)$ & $63(94)$ & & 67 \\
\hline No & $17(6,2)$ & $258(93,8)$ & & $23(8,4)$ & $252(91,6)$ & & 275 \\
\hline Sexo & & & 0,583 & & & 0,160 & \\
\hline Masculino & $9(6,7)$ & $125(93,3)$ & & $14(10,4)$ & $120(89,6)$ & & 134 \\
\hline Femenino & $11(5,3)$ & $197(94,7)$ & & $13(6,3)$ & $195(93,8)$ & & 208 \\
\hline Edad (años) & & & 0,019 & & & 0,196 & \\
\hline $15-20$ & $1(7,1)$ & $13(92,9)$ & & $1(7,1)$ & $13(92,9)$ & & 14 \\
\hline $21-40$ & $3(3,1)$ & $94(96,9)$ & & $5(5,2)$ & $92(94,8)$ & & 97 \\
\hline $41-60$ & $4(4,1)$ & $94(95,9)$ & & $5(5,1)$ & $93(94,9)$ & & 98 \\
\hline $61-80$ & $8(6,9)$ & $108(93,1)$ & & $13(11,2)$ & $103(8,8)$ & & 116 \\
\hline$>80$ & $4(23,5)$ & $13(76,5)$ & & $3(17,6)$ & $14(82,4)$ & & 17 \\
\hline Cirugía abdominal previa & & & 0,547 & & & 0,883 & \\
\hline Sí & $2(8,7)$ & $21(91,3)$ & & $2(8,7)$ & $21(91,3)$ & & 23 \\
\hline No & $18(5,6)$ & $301(94,4)$ & & $25(7,8)$ & $294(92,2)$ & & 319 \\
\hline Comorbilidad & & & 0,042 & & & 0,005 & \\
\hline Sí & $9(10,2)$ & $79(89,8)$ & & $13(14,8)$ & $75(85,2)$ & & 88 \\
\hline No & $11(4,3)$ & $243(95,7)$ & & $14(5,5)$ & $240(94,5)$ & & 254 \\
\hline ASA & & & 0,627 & & & 0,270 & \\
\hline 1 & $3(3,3)$ & $89(96,7)$ & & $3(3,3)$ & $89(96,7)$ & & 92 \\
\hline II & $14(6,6)$ & $198(93,4)$ & & $20(9,4)$ & $192(90,6)$ & & 212 \\
\hline III & $3(8,1)$ & $34(91,9)$ & & $4(10,8)$ & $33(89,2)$ & & 37 \\
\hline IV & $0(0)$ & $1(100)$ & & $0(0)$ & $1(100)$ & & 1 \\
\hline IMC & & & 0,822 & & & 0,408 & \\
\hline Bajo peso & $0(0)$ & $3(100)$ & & $0(0)$ & $3(100)$ & & 3 \\
\hline Peso normal & $10(7)$ & $132(93)$ & & $15(10,6)$ & $127(89,4)$ & & 142 \\
\hline Sobrepeso & $6(4,6)$ & $124(95,4)$ & & $9(6,9)$ & $121(93,1)$ & & 130 \\
\hline Obesidad & $4(6)$ & $63(94)$ & & $3(4,5)$ & $64(95,5)$ & & 67 \\
\hline
\end{tabular}




\begin{tabular}{|c|c|c|c|c|c|c|c|}
\hline \multicolumn{2}{|l|}{ Colecistitis } & \multicolumn{3}{|c|}{0,010} & \multicolumn{3}{|c|}{0,001} \\
\hline No & $2(1,6)$ & $125(98,4)$ & & $2(1,6)$ & $125(98,4)$ & & 127 \\
\hline Sí & $18(8,4)$ & $197(91,6)$ & & $25(11,6)$ & $190(88,4)$ & & 215 \\
\hline Grado 1 & $7(5,9)$ & $111(94,1)$ & 0,023 & $11(9,3)$ & $107(90,7)$ & 0,005 & 118 \\
\hline Grado 2 & $10(11,2)$ & $79(88,8)$ & & $13(14,6)$ & $76(85,4)$ & & 89 \\
\hline Grado 3 & $1(12,5)$ & $7(87,5)$ & & $1(12,5)$ & $7(87,5)$ & & 8 \\
\hline \multicolumn{2}{|l|}{ Colangitis } & \multicolumn{3}{|c|}{0,198} & \multicolumn{3}{|c|}{0,474} \\
\hline No & $17(5,4)$ & $299(94,6)$ & & $24(7,6)$ & $292(92,4)$ & & 316 \\
\hline Sí & $3(11,5)$ & $23(88,5)$ & & $3(11,5)$ & $23(88,5)$ & & 26 \\
\hline Grado 1 & $2(20)$ & $8(80)$ & 0,541 & $2(20)$ & $8(80)$ & 0,541 & 10 \\
\hline Grado 2 & $1(7,1)$ & $13(92,9)$ & & $1(7,1)$ & $13(92,9)$ & & 14 \\
\hline Grado 3 & $0(0)$ & $2(100)$ & & $0(0)$ & $2(100)$ & & 2 \\
\hline \multicolumn{2}{|l|}{ Pancreatitis } & \multicolumn{3}{|c|}{0,120} & \multicolumn{3}{|c|}{0,068} \\
\hline No & $20(6,5)$ & $287(93,5)$ & & $27(8,8)$ & $280(91,2)$ & & 307 \\
\hline Sí & $0(0)$ & $35(100)$ & & $0(0)$ & $35(100)$ & & 35 \\
\hline Leve & $0(0)$ & $29(100)$ & 0,298 & $0(0)$ & $29(100)$ & 0,188 & 29 \\
\hline Moderadamente grave & $0(0)$ & $6(100)$ & & $0(0)$ & $6(100)$ & & 6 \\
\hline
\end{tabular}

CPRE: colangiopancreatografía retrógrada endoscópica; ASA: clasificación de la American Society of Anesthesiologists; IMC: índice de masa corporal

biliar). De los 67 pacientes el grupo 'con CPRE', tres pacientes tuvieron colección intraabdominal $\mathrm{y}$ un paciente tuvo una crisis hipertensiva, para un total de $4(6 \%)$ complicaciones posoperatorias en este grupo.

La incidencia global de lesión de la vía biliar fue de 0,9\%: ninguna en el grupo 'con CPRE' y tres en el grupo 'sin CPRE'.

En el grupo 'con CPRE', la mediana de estancia hospitalaria fue de 3 días (rango: I-I6) y la de la estancia en la unidad de cuidado intensivo fue de 5 días (rango: 4-IO); en el grupo 'sin CPRE' fueron de 2 días (rango: 0-30) y de 5 días (rango: I-I8), respectivamente.

La mortalidad global fue de $0,3 \%$, pues solo falleció un paciente. Pertenecía al grupo 'sin CPRE', tenía un diagnóstico prequirúrgico de colecistitis aguda grado 2 , con comorbilidades de hipertensión arterial sistémica, enfermedad pulmonar obstructiva crónica y diabetes mellitus, y su cirugía no fue descrita ni clasificada como difícil. En el posoperatorio, presentó descompensación de la diabetes y falla respiratoria. De un paciente se desconoce el resultado, ya que fue remitido a la unidad de cuidados intensivos coronarios de otra institución.
Se elaboró un modelo de regresión logística binaria para establecer la asociación entre CPRE y colecistectomía difícil. En el modelo se incluyeron las variables que pudieran influir en la dificultad técnica de la colecistectomía laparoscópica, según los resultados descritos y la literatura. Las variables que resultaron significativas fueron: edad, sexo masculino, cirugía abdominal previa, colecistitis aguda y mayor gravedad de la colecistitis aguda (tabla 5). La CPRE previa no tuvo relación con dificultad en la colecistectomía.

\section{Discusión}

En el año 20I7 en Popayán, era más fácil acceder a una colangiopancreatografía retrógrada endoscópica que a la colangiorresonancia o la ecoendoscopia, por lo que se optaba por usarla para el diagnóstico y el tratamiento de los pacientes con sospecha o diagnóstico de coledocolitiasis. En la actualidad, tenemos acceso a la ecoendoscopia como apoyo diagnóstico en estos casos.

De las 342 colecistectomías laparoscópicas incluidas el estudio, el 45,3\% (I55), cumplieron los criterios como cirugía difícil; Singh, et al., reportaron una tasa de 2I, $5 \%$ colecistectomías difíciles en su cohorte de II años ${ }^{7}$, Fruscione, et al., una 
Tabla 5. Variables asociadas a mayor probabilidad de colecistectomía laparoscópica difícil según la regresión logística binaria.

\begin{tabular}{llcc}
\hline Variable & OR & IC $_{95 \%}$ & $\mathbf{p}$ \\
\hline Sexo masculino & 2,2 & $1,32-3,62$ & 0,002 \\
Edad & 1,73 & $1,31-2,27$ & 0,000 \\
Cirugía abdominal previa & 2,69 & $1,04-6,93$ & 0,041 \\
Colecistitis aguda & 8,6 & $7,04-10,5$ & 0,001 \\
Gravedad de la colecistitis & 2,66 & $1,5-4,7$ & 0,001 \\
\hline
\end{tabular}

de $18, \mathrm{I} \%{ }^{\mathrm{IO}} \mathrm{y}$, Abdelrahim, una de $\mathrm{I} 3 \%{ }^{\mathrm{II}}$. Nuestra proporción duplica y, en algunos casos triplica, la descrita en la literatura; esto refleja la complejidad de la enfermedad biliar en la población caucana. Llama la atención que, solo el 45,2 \% de las colecistectomías clasificadas como difíciles fueron consideradas así por los cirujanos en la descripción quirúrgica, probablemente, porque la mayoría de los cirujanos generales tienen más de Io años de experiencia y están frecuentemente expuestos a la enfermedad biliar compleja, lo que hace que tiendan a sentirse cómodos con el procedimiento, incluso, con aquellos difíciles.

En algunas publicaciones, se ha mencionado que la colecistectomía laparoscópica tiene un mayor riesgo de complicaciones después de la CPRE, con tiempo quirúrgico más prolongado, mayor sangrado y mayor tasa de conversión a cirugía abierta ${ }^{4}$. Se han reportado tasas de conversión del 8,3\% en casos con esfinterotomía previa, comparadas con 3,4 \% para las colecistectomías estándar ${ }^{3}$. En el presente estudio, la CPRE previa, la papilotomía o la colocación de endoprótesis biliar, no se asociaron con dificultad durante la colecistectomía laparoscópica, ni con conversión a cirugía abierta, mayor tiempo quirúrgico, reintervención, prolongación de la estancia hospitalaria o complicaciones posoperatorias.

Estos resultados no son aislados y concuerdan con los de estudios de mayor peso estadístico. En una revisión sistemática de I4 estudios y I.930 pacientes, Friis, et al. ${ }^{\mathrm{I}}$, encontraron un aumento de la tasa de conversión a cirugía abierta de 4,2 \% cuando la colecistectomía laparoscópi- ca se practicó dentro de las 24 horas después de la CPRE, de 7,6\%, cuando fue de 24 a 72 horas después, de $12,3 \%$ cuando fue de 72 horas a dos semanas después, de $12,3 \%$ cuando fue de 2 a 6 semanas después, y de I $4 \%$ cuando la operación se retrasó más de seis semanas. Además, concluyeron que la colecistectomía laparoscópica temprana después de una CPRE no aumenta la mortalidad, las complicaciones perioperatorias, ni la estancia hospitalaria y, por el contrario, reduce el riesgo de recurrencia y progresión de la enfermedad, que se presentan cuando ese lapso de tiempo se prolonga.

Una revisión sistemática de Cochrane ${ }^{13}$ mostró que los pacientes en los que se postergaba la colecistectomía laparoscópica después de la CPRE, con una conducta de "esperar y observar", tuvieron tasas mayores de dolor biliar recurrente (riesgo relativo, $\mathrm{RR}=\mathrm{I} 4,56 ; \mathrm{IC}_{95} \% 4,95-42,78$; $\mathrm{p}=<\mathrm{OOOOOI})$, de ictericia o colangitis $\left(\mathrm{RR}=2,53 ; \mathrm{IC}_{95} \%\right.$ I,09-5,87; $\mathrm{p}=0,03)$ y de repetición de la CPRE u otra modalidad de colangiografía $\left(\mathrm{RR}=2,36 ; \mathrm{IC}_{95 \%}\right.$ I,29-4,32; $p=0,005)$. Teniendo en cuenta estos datos y los presentes resultados, en los pacientes que ingresan por urgencias consideramos seguro, y además necesario, hacer una colecistectomía laparoscópica lo más pronto posible después de la realización de la CPRE por enfermedad biliar.

Dado que en algunos miembros del equipo quirúrgico existía la impresión de que eran más difíciles las colecistectomías después de una CPRE, también se compararon las cirugías reportadas por los cirujanos como difíciles, según se hubiera realizado o no el procedimiento endoscópico antes de la cirugía. Se encontró que el $22 \%$ de las colecistectomías laparoscópicas del grupo 'con CPRE' y el $20 \%$ de las colecistectomías del grupo 'sin CPRE', fueron reportadas en la descripción quirúrgica como difíciles. De esto se puede inferir que, la CPRE previa a la colecistectomía laparoscópica tampoco influyó en la percepción intraoperatoria de dificultad técnica por parte de los cirujanos.

La tasa de conversión de colecistectomía laparoscópica a abierta a menudo se encuentra entre el 5 y el $7 \%$. Abdelrahim, et al. II, reportaron una 
proporción de $13 \%$ de colecistectomías difíciles, con una tasa de conversión del 5,5\%, mientras Aldana, en un hospital de IV nivel en Colombia, encontró una tasa de conversión de 6,I \% en cirugías urgentes y de I,I \% en las programadas ${ }^{14}$. En el presente estudio, la tasa global de conversión fue de $5,5 \%$, similar a la reportada en la literatura. El sexo masculino se asoció significativamente con la conversión a cirugía abierta y, además, la mayoría de los pacientes que la requirieron eran mayores de 40 años; solo una colecistectomía laparoscópica fue convertida en un paciente menor de 40 años $(\mathrm{p}=0,092)$.

Quienes apoyan que la colecistectomía laparoscópica es más difícil después de una CPRE creen que esto se debe a la inflamación y posterior fibrosis de las estructuras del ligamento hepatoduodenal por la instrumentación de la vía biliar, como efecto directo del medio de contraste o por la colonización bacteriana de la vía biliar posterior a la papilotomía, lo cual dificulta la disección del triángulo de Calot ${ }^{4}$. Por las causas de conversión encontradas en nuestros pacientes, en quienes el Ioo \% de las colecistectomías laparoscópicas que se convirtieron a cirugía abierta en el grupo 'con CPRE' lo hicieron por difícil disección o falta de visualización de las estructuras del triángulo de Calot, en comparación con $8,3 \%$ en el grupo 'sin CPRE', en el que la conversión se debió con mayor frecuencia a plastrón o sangrado, deducimos que, en los pacientes con el antecedente de CPRE en los que el procedimiento es laborioso, esto sí se debe a inflamación y fibrosis del triángulo de Calot; aunque esto no sucede en todos y, en nuestro medio, existen otras variables que tienen mayor peso para generar dificultades técnicas o la conversión a cirugía abierta de la colecistectomía laparoscópica.

Las variables que se asociaron de forma estadísticamente significativa con una colecistectomía laparoscópica difícil en el análisis bivariado fueron sexo masculino, edad, colecistitis aguda y gravedad de la colecistitis aguda. Estos factores de riesgo ya se han mencionado ampliamente en la literatura sobre colecistectomía difícil, y como predictores de complicaciones y de conversión a cirugía abierta $^{\mathrm{I}, \mathrm{IO}, \mathrm{I} 4}$.

El análisis bivariado también reveló asociación entre la edad, la presencia de alguna comorbilidad, el diagnóstico prequirúrgico de colecistitis aguda y una mayor gravedad de la colecistitis aguda, con una reintervención después de las 24 horas. Las complicaciones posoperatorias fueron más frecuentes en pacientes con comorbilidad, colecistitis aguda y mayor gravedad de la colecistitis aguda.

Para tratar de establecer una verdadera asociación causal y determinar la probabilidad de cirugía difícil con la presencia de estos factores, se utilizó un modelo de regresión logística, donde se obtuvieron como factores predictores para colecistectomía laparoscópica difícil, mayor edad, sexo masculino, cirugía abdominal previa, colecistitis aguda y mayor gravedad de la colecistitis aguda.

Se hizo una búsqueda para intentar explicar por qué estos factores hacen más difícil la colecistectomía laparoscópica. Aldana, et al. ${ }^{14}$, encontraron el antecedente de cirugía abdominal como predictor de complicaciones de colecistectomía por laparoscopia y describieron el antecedente ligado a mayores dificultades técnicas, tanto para el ingreso de los trocares como para el procedimiento mismo, dada la presencia de adherencias en la cavidad abdominal.

Efectivamente, las colecistectomías laparoscópicas efectuadas durante un episodio de colecistitis aguda son más difíciles, las lesiones del colédoco aumentan proporcionalmente con el grado de inflamación y, si se presenta inflamación aguda concomitantemente con inflamación crónica, el grado de dificultad es mayor. Álvarez, et al., mencionan que la inflamación hace que el cirujano se confunda fácilmente e interprete erróneamente la anatomía ${ }^{15}$.

En cuanto al mayor grado de dificultad y las complicaciones en el sexo masculino, quizás esto se deba a mayor fibrosis y cambios anatómicos por inflamación. Akcayaca, et al. ${ }^{16}$, encontraron mayor frecuencia de adherencias entre la vesícula biliar, y el epiplón y otros órganos, en pacientes masculinos sometidos a colecistecto- 
mía laparoscópica; además, tanto los cambios inflamatorios agudos como los crónicos, fueron más prominentes en las colecistectomías de pacientes masculinos que en las de las mujeres. El mismo grupo menciona que los hombres son más negligentes con respecto a su salud y buscan atención médica más tarde, por lo que se presentan con cambios inflamatorios crónicos y agudos más graves en su vesícula biliar. También, se han encontrado niveles más altos de colágeno e hidroxiprolina en muestras de colecistectomía de pacientes varones, lo que sugiere una diferencia en los procesos celulares de inflamación ${ }^{17}$.

La mortalidad en nuestra cohorte fue de $0,3 \%$, lo esperado según lo reportado por otros autores ${ }^{14}$.

Como conclusión de este trabajo, creemos que los resultados son valiosos ya que se puede inferir que, en nuestro medio y en pacientes no ambulatorios, es seguro practicar la colecistectomía laparoscópica temprana después de una CPRE. Además, existen otras variables que predicen una colecistectomía difícil de forma significativa, las cuales se deben tener en cuenta a la hora de planificar la cirugía. La colecistitis aguda y una mayor severidad de esta fueron las variables que tuvieron relación estadísticamente significativa con la mayoría de los resultados estudiados, como cirugía difícil, mayor estancia hospitalaria y mayor estancia en la unidad de cuidado intensivo. Por tales razones, deberían discutirse con mucha más precaución las diferentes opciones de manejo a la hora de abordar estos pacientes, tomando precauciones adicionales para prevenir complicaciones, como asignar a los miembros del equipo con más experiencia y habilidad quirúrgica para el manejo de esos casos.

\section{Cumplimiento de normas éticas}

Consentimiento informado: El estudio fue aprobado por el Comité de Ética Médica del Hospital Universitario San José; dado que el trabajo se basa en la revisión de historias clínicas y no hay ninguna intervención por parte de los investigadores en los pacientes, no se requirió de consentimiento informado.

\section{Conflicto de interés. Ninguno}

Fuentes de financiación: Autofinanciado

\section{Referencias}

I. Bourgouin S, Mancini J, Monchal T, Calvary R, Bordes J, Balandraud P. How to predict difficult laparoscopic cholecystectomy? Proposal for a simple preoperative scoring system. Am J Surg. 20I6;212:873-8I. https://doi.org/Io.IoI6/j.amjsurg.20I6.04.003

2. Reinders JSK, Goud A, Timmer R, Kruyt PM, Witteman BJM, Smakman N, et al. Early laparoscopic cholecystectomy improves outcomes after endoscopic sphincterotomy for choledochocystolithiasis. Gastroenterology. 2010;138:2315-20.

https://doi.org/I0.I053/j.gastro.2010.02.052

3. Sarli L, Lusco D, Roncoroni L. Preoperative endoscopic sphincterotomy and laparoscopic cholecystectomy for the management of cholecystocholedocholithiasis: IOyear experience. World J Surg. 2003;27: 180-6. https://doi.org/IO.IO07/s00268-002-6456-8

4. Mann K, Belgaumkar AP, Singh S. Post-endoscopic retrograde cholangiography laparoscopic cholecystectomy: Challenging but safe. JSLS. 20I3;17:37I-5. https://doi.org/I0.4293/I086808I3XI36547545355II

5. Yokoe M, Takada T, Strasberg SM, Solomkin JS, Mayumi T, Gomi H, et al. TGI3 diagnostic criteria and severity grading of acute cholecystitis (with videos). J Hepatobiliary Pancreat Sci. 2013:20:35-46. https://doi.org/IO.IO07/s00534-0I2-0568-9

6. Banks PA, Bollen TL, Dervenis C, Gooszen HG, Johnson CD, Sarr MG, et al, Acute Pancreatitis Classification Working Group. Classification of acute pancreatitis-2012: revision of the Atlanta classification and definitions by international consensus. Gut 2013;62:IO2-II. https://doi.org/IO.II36/gutjnl-2012-302779

7. Singh K, Ohri A. Laparoscopic cholecystectomy - Is there a need to convert?. J Minimal Access Surg. 2005;1:59-62. https://doi.org/I0.4I03/0972-994I.I6528

8. Sinha R. Difficult laparoscopic cholecystectomy - When and where is the need to convert?. Apollo Medicine. 2010;7:I35-7. https://doi.org/I0.IoI6/So976-00I6(II)60095-I

9. American Society for Gastrointestinal Endoscopy. The role of endoscopy in the evaluation of suspected choledocholithiasis. Gastrointestinal Endoscopy. 2010;71:I-9. https://doi.org/Io.IoI6/j.gie.2009.09.04I

Io. Fruscione M, Kirks RC, Cochran A, Murphy K, Baker $\mathrm{EH}$, Martinie JB, et al. Routine versus difficult chole- 
cystectomy: Using predictive analytics to assess patient outcomes. HPB (Oxford). 2019;2I:77-86.

https://doi.org/IO.IOI6/j.hpb.2018.06.I805

II. Abdelrahim WE, Elsiddig KE, Wahab AA, Saad M, Saeed H, Khalil EAG. Subtotal laparoscopic cholecystectomy influences the rate of conversion in patients with difficult laparoscopic cholecystectomy: Case series. Ann Med Surg. 20I7;19:19-22.

https://doi.org/IO.IOI6/j.amsu.2017.04.0I8

I2. Friis C, Rothman JP, Burcharth J, Rosenberg J. Optimal timing for laparoscopic cholecystectomy after endoscopic retrograde cholangiopancreatography: A systematic review. Scand J Surg. 2018;107:99-IO6.

https://doi.org/Io.II77/I4574969I7748224

13. McAlister VC, Davenport E, Renouf E. Postergación de la colecistectomía en pacientes con esfinterotomía endoscópica (Revisión Cochrane traducida). En: La Biblioteca Cochrane Plus, 2008 Número 4. Oxford: Update Sof- tware Ltd.; 2008. Fecha de consulta: I8 de julio de 2018. Disponible en: http://www.bibliotecacochrane.com

I4. Aldana GE, Martínez LE, Hosman MA, Ardila DA, Mariño IF, Sagra MR, et al. Factores predictores perioperatorios de complicaciones de la colecistectomía por laparoscopia. Rev Colomb Cir. 20I8;33:I62-72. https://doi.org/IO.30944/20II7582.58

I5. Álvarez LF, Rivera D, Esmeral ME, García MC, Toro DF, Rojas OL. Colecistectomía laparoscópica difícil, estrategias de manejo. Rev Colomb Cir. 2013;28:186-95.

I6. Akcakaya A, Okan I, Bas G, Sahin G, Sahin M. Does the difficulty of laparoscopic cholecystectomy differ between genders? Indian J Surg. 2015;77:452-6. https://doi.org/IO.I007/sI2262-013-0872-X

I7. Yol S, Kartal A, Vatansev C, Aksoy F, Toy H. Sex as a factor in conversion from laparoscopic cholecystectomy to open surgery. JSLS. 2006;10:359-63. 